\section{Prevalence and antibiotic susceptibility profile of methicillin resistant Staphylococcus aureus in Accra, Ghana}

\author{
Stephen T. Odonkor, ${ }^{1}$ Mercy J. Newman, ${ }^{2}$ \\ Kennedy K. Addo ${ }^{3}$ \\ ${ }^{1}$ Radiological and Medical Sciences \\ Research Institute, Ghana Atomic Energy \\ Commission; ${ }^{2}$ Department of \\ Microbiology, University of Ghana \\ Medical School; ${ }^{3}$ Department of \\ Bacteriology, Noguchi Memorial Institute \\ for Medical Research, University of \\ Ghana, Accra, Ghana
}

\section{Abstract}

Over the last four decades, methicillin-resistant Staphylococcus aureus (MRSA) has spread throughout the world and become highly endemic in many geographical areas. This pathogen causes severe morbidity and mortality in hospitals worldwide. MRSA is also considered a major community acquired pathogen throughout the world. MRSA is implicated in serious clinical conditions such as bacteremia, pneumonia, and intra-abdominal infection. The objective of this study was to determine the prevalence of MRSA in Accra, Ghana, and also to determine its antibiotic susceptibility profile. Two hundred and fifty Staphylococcus aureus isolates from routine microbiological specimens were collected from five hospitals in Accra. MRSA screening assay was used to screen for MRSA. Disc diffusion method (KirbyBauer) was used to determine the susceptibility of the MRSA. The MRSA screening assay, which is very close to the polymerase chain reaction in terms of specificity and sensitivity, showed that 84 of the 250 isolates were MRSA, giving a prevalence rate of $33.6 \%$. MRSA strains were susceptible to erythromycin; 63 out of the 84 MRSA isolates were susceptible representing $75 \%$. This was followed by gentamicin 46 (54.7\%), cotrimoxazole $35(49 \%)$, cefuroxime 33 (38\%), flucloxacillin 24 (28.6\%), and ampicillin 13 (15.5\%). Penicillin 4 (4.8\%) and tetracycline 6 (7.1\%) were the least susceptible. The findings from this study emphasize the need for continual surveillance of MRSA and of antibiotic resistance in general.

\section{Introduction}

Methicillin-resistant Staphylococcus aureus
(MRSA) is considered a major nosocomial and community acquired pathogen throughout the world. MRSA is implicated in serious clinical conditions such as bacteremia, pneumonia, and intra-abdominal infection. ${ }^{1}$ Staphylococcus aureus strains resistant to methicillin have become a well-known etiological agent in a wide variety of infections. These infections have become a common problem in hospitals and in the community, and have been associated with prolonged hospital stay and increased hospital costs.

Moreover, few therapeutic options are available to treat infected patients. ${ }^{1,2}$

One of the earliest reports on MRSA in Africa was by Scragg et $a l^{3}$ Subsequently, MRSA was reported from the mid ' 80 s to the 1990 s in a number of African countries including Ethiopia, ${ }^{4}$ Kenya, ${ }^{5}$ Nigeria, ${ }^{6,7}$ Senegal, ${ }^{8}$ South Africa and Sudan. ${ }^{9-12}$ Resistance to methicillin (and to all $\beta$-lactam antibiotics) in Staphylococcus aureus is primarily associated with the acquisition of the mecA gene coding for the penicillin-binding protein 2a (PBP 2a), involved in bacterial cell wall synthesis. ${ }^{2} \mathrm{~A}$ distinctive feature of methicillin resistance is its heterogeneous nature, ${ }^{13,14}$ with the level of resistance varying according to the culture conditions and $\beta$-lactam antibiotic being used. The majority of cells in heterogeneous strains (typically $99.9 \%$ or more) are susceptible to low concentrations of $\beta$-lactam antibiotics, e.g. 1 to $5 \mu \mathrm{g} / \mathrm{mL}$ of methicillin. ${ }^{14}$

The detection of methicillin resistance, however, is complicated by the fact that its phenotypic expression in many strains is heterogeneous. ${ }^{15}$ This has resulted in the development of various laboratory techniques to enhance the expression of this resistance in vitro. ${ }^{3,4}$ Examples of these methods are slide latex agglutination test, rapid solid-phase immunoassay, and various disc diffusion methods. mecA gene detection tests based on polymerase chain reaction (PCR) or DNA hybridization have proved to be more specific and more sensitive than conventional tests, particularly in very heterogeneous strains. ${ }^{5}$ Little work has been done on MRSA in Ghana. The objective of this study was to determine the prevalence and antibiogram of MRSA in Accra, Ghana.

\section{Materials and Methods}

\section{Clinical specimen}

Two hundred and fifty (250) Staphylococcus aureus isolates from routine microbiological specimens were collected from five hospitals in Accra. Categories of patients included those hospitalized with suspected clinical infections (in-patients) and those attending the outpa-
Correspondence: Stephen T. Odonkor, Radiological and Medical Sciences Research Institute (RAMSRI), Ghana Atomic Energy Commission. P. 0. Box LG 80, Legon-Accra, Ghana.

Tel. +233.20.986221- Fax: +233.30.2400807

E-mail: stodonkor@yahoo.co.uk

Key words: methicillin resistant Staphylococcus aureus (MRSA), mecA gene, penicillin-binding protein 2a (PBP 2a), $\beta$-lactam, antibiotics.

Acknowledgements: the authors are grateful to all the biomedical scientists in hospitals in Accra where isolates were obtained.

Contributions: MJN, STO, conception and design; STO, data gathering, manuscript writing; KKA, supervision and coordination of laboratory analysis.

Conflict of interests: the authors report no potential conflict of interests.

Received for publication: 29 August 2011.

Revision received: 9 December 2011.

Accepted for publication: 8 February 2012.

This work is licensed under a Creative Commons Attribution NonCommercial 3.0 License (CC BYNC 3.0).

(C) Copyright S.T. Odonkor et al., 2012

Licensee PAGEPress, Italy

Microbiology Research 2012; 3:e20

doi:10.4081/mr.2012.e20

tients' department for the first time without hospitalization (out-patients). Only one isolate was recovered from each patient, presenting with a particular case. The $S$. aureus isolates were obtained from cultures of different specimens including wounds, sputum, blood, aspirates, urine, and cerebrospinal fluids. These isolates were collected and sub-cultured on slopes of Mueller Hinton agar for storage.

\section{Patient bio-data}

Full patient bio-data such as age, sex, and types of infection was obtained from request forms.

\section{Culture}

Suspected colonies of S. aureus from primary culture plates of Blood agar, Chocolate agar and MacConkey agar were confirmed as S. aureus, by Gram Reaction, positive catalase, Tube coagulase and Deoxyribonucleases (DNAse) test. MRSA screening assay was used to screen for Methicillin resistance. The MRSA- screen assay is a 5-minutes slide latex agglutination test based on detection of PBP2a. The method involves extraction of PBP2a from suspensions of colonies and detection by agglutination with latex particles coated with monoclonal antibodies to PBP2a. Inocula for susceptibility testing 
were made using suspensions from over nightcultures of MRSA on Müller-Hinton agar. An inoculum equivalent to 0.5 McFarland $\left(10^{8}\right.$ $\mathrm{CFU} / \mathrm{mL}$ ) turbidity standard was used for each test. Disc diffusion method (Kirby-Bauer method) was used to determine the susceptibility of the MRSA, to commonly used antimicrobial agents. These included: Penicillin (1 ug), Ampicillin (10 ug), Flucloxacillin (5 ug), Erythromycin (5 ug), Tetracycline (10 ug), Cotrimoxazole (25 ug), Cefuroxime (30 ug), Gentamicin (10 ug). The inoculum was compared to a 0.5 McFarland standard and the control was MRSA (ATCC 6571).

\section{Results}

From Table 1, a total number of 84 isolates from $250 \mathrm{~S}$. aureus isolates tested positive for MRSA. Out of this, 87 MRSA wound specimen produced the highest (46) of MRSA representing $54.7 \%$ of total MRSA detected. This is followed by isolates specimen from swabs representing $21.4 \%$ of MRSA isolated. Blood specimen is the third highest (14.3\%) source of MRSA. This is followed by Aspirates (3.6\%), Sputum (2.4\%) and Cerebrospinal fluid (2.4\%). Urine specimen was the least only with 1 specimen representing (1.2\%) tested positive for MRSA.

Figure 1 shows the percentage of MRSA and susceptible Staphylococcus aureus (MSSA). The prevalence of MRSA from this study is therefore $33.6 \%$.

\section{Distribution of methicillin-resistant Staphylococcus aureus isolates}

Table 2 shows the distribution of 84 MRSA isolates recovered in terms of out-patients and in-patients. In patients had the highest carriage of MRSA representing $69 \%$, whiles out patients had a total of 26 isolates representing $31 \%$. No MRSA (0\%) was detected from sputum, aspirates, cerebrospinal fluids and urine specimen recovered from out patients.

\section{Discussion}

Undoubtedly the epidemiology of MRSA has continued to evolve since its first appearance more than three decades ago. Initially there were sporadic reports of methicillin resistance amongst nosocomial $S$. aureus isolates. But later MRSA became a well established hospital acquired pathogen with few reports of community acquired isolates. Recent studies report of increases of prevalence of MRSA. In this present study (Figure 1) $33.6 \%$ of $S$. aureus isolates

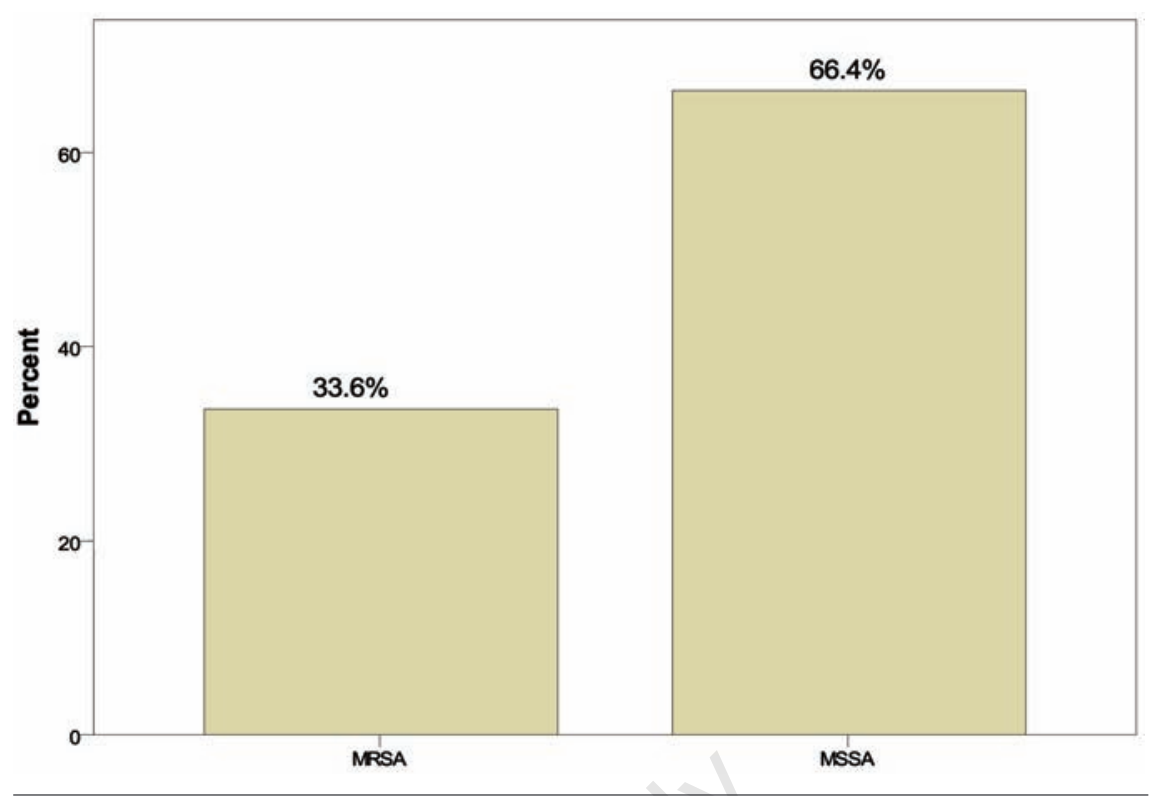

Figure 1. Percentage of methicillin-resistant Staphylococcus aureus isolated from clinical specimen.

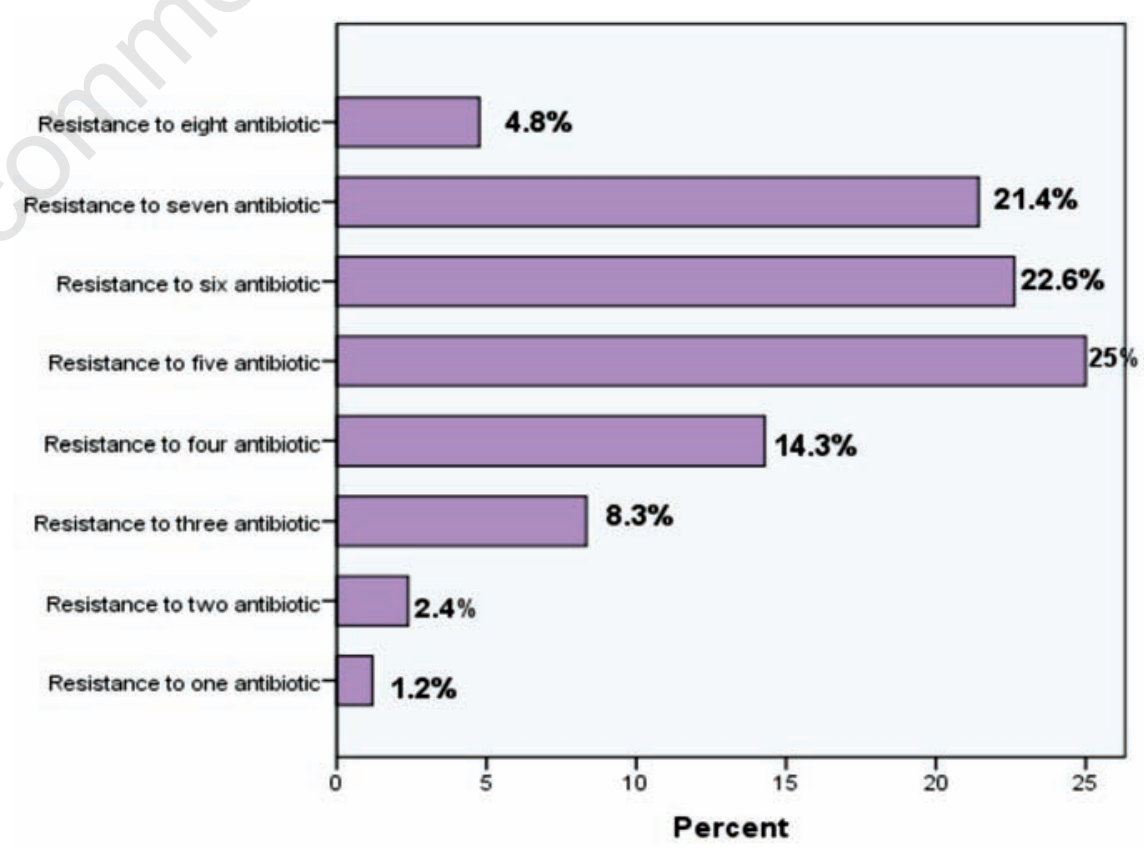

Figure 2. Multidrug resistance of methicillin-resistant Staphylococcus aureus. Multi drug resistance of methicillin-resistant Staphylococcus aureus. Generally, out of the 84 MRSA isolates, $25 \%$ were resistant to more than five antibiotics used, followed by $22.6 \%, 21.4 \%$, $14.3 \%, 8.3 \%$ and $4.8 \%$ for six antibiotics, seven antibiotics, four antibiotics, three antibiotics and eight antibiotics respectively. Also $2.4 \%$ of MRSA were resistant to two antibiotics. Whiles $1.3 \%$ were resistant to one antibiotic. In general $96.3 \%$ of the isolates were resistant to three or more antibiotics. 
were found to be resistant to methicillin, whereas in the previous studies done in Accra, prevalence was found to be $22 \%$ in 2000 and 31.5\% in 2002 (see Safo-Kantaka A, 2000. The studies of Methicillin resistant Staphylococcus aureus (MRSA) in Korle-Bu Teaching Hospital. Personal communication). This implies that the incidence of MRSA keeps changing every year and is on a rise as compared to the last few years.

The MRSA screen assay detected 84 of the $250 \mathrm{~S}$. aureus isolates as MRSA (Table 1). No agglutination occurred when the 250 isolates were tested with the negative-control latex. The MRSA PBP2a latex agglutination screen assay is latex particles sensitized with anticlonal antibody against PBP2a and react specifically with MRSA to cause agglutination. It is reported to have $97.6 \%$ sensitivity and it distinguishes between very low level MRSA from MSSA. ${ }^{16,17}$ Bowers et al., reported MRSAlatex agglutination test as a reliable and rapid detection test from both broth pure culture and selective media as well as being a reliable alternative to mec A PCR for the definite diagnosis of MRSA. ${ }^{18}$ Interestingly, Atoum et al. reported strains of negative mecA being methicillin resistant and positive mecA being methicillin sensitive. These observations are explained as being due to non functional mecA gene or non active PBP2a protein. Consequently, they recommended the use of a combination of both molecular and microbiological methods for detection of MRSA. ${ }^{19}$ However PBP2a latex agglutination has demonstrated $100 \%$ agreement for both mecA- positive and negative strains. ${ }^{20}$

It is well established that the major mechanism of resistance to methicillin and other antistaphyloccoccal penicilins in $S$. aureus involves the production of an additional membrane penicillin-biding protein. In adtion to the PBP2a production, most methicilin-resistant $S$. aureus also produce $\beta$-lactamases. ${ }^{21}$ There is however, limited information about the relationship between B-lactamase(s) of MRSA and the four B-lactamases (types A, B, C and D) produce by methicillin susceptible strains of $S$. aureus.

Most of the MRSA strains (34.8\%) in this study came from wound and skin swabs (Table 2). This is similar to the study by Felten et al, in which it was reported that MSSA from skin lesions probably acquired the mecA gene by horizontal transfer from other skin Staphylo coccus species. ${ }^{1}$

MRSA is generally known to be resistant to $\beta$-lactam antibiotics (Figure 2). However penicillin, ampicillin and flucloxacillin (all $\beta$-lactam antibiotics) were included in the study to ascertain their antibiotic susceptibility patterns. From Table 3 penicillin had the highest resistance of $92.2 \%$ followed by ampicillin $84.5 \%$ and flucloxacillin $71.4 \%$. These findings
Table 1. Isolates of methicillin-resistant Staphylococcus aureus from different clinical specimen.

\begin{tabular}{lccc} 
Clinical & $\begin{array}{c}\text { No. of } S \text {. aureus } \\
n=250\end{array}$ & $\begin{array}{c}\text { No. of MRSA } \\
\mathbf{n}=84\end{array}$ & \% MRSA \\
Wound & 87 & 46 & 54.7 \\
Swabs & 55 & 18 & 21.4 \\
\hline Blood & 27 & 12 & 14.3 \\
Aspirates & 30 & 3 & 3.6 \\
\hline Sputum & 25 & 2 & 2.4 \\
Cerebrospinal fluid & 17 & 2 & 2.4 \\
Urine & 9 & 1 & 1.2 \\
Total & 250 & 84 & 100
\end{tabular}

A total number of 84 isolates from 250 S. aureus isolates tested positive for methicillin-resistant Staphylococcus aureus (MRSA). Out of this, 87 MRSA wound specimen produced the highest (46) of MRSA representing $54.7 \%$ of total MRSA detected. This is followed by specimen from swabs representing $21.4 \%$ of MRSA isolated. Blood specimen is the third highest (14.3\%) source of MRSA. This is followed by Aspirates $(3.6 \%)$, Sputum (2.4\%) and Cerebrospinal fluid (2.4\%). Urine specimen was the least only with 1speceimen representing (1.2\%) tested positive for MRSA.

Table 2. Distribution of methicillin-resistant Staphylococcus aureus isolates (outpatients and inpatient).

\begin{tabular}{lccc} 
Clinical Specimen & $\begin{array}{c}\text { No. of MRSA } \\
\mathbf{n}=(84)\end{array}$ & $\begin{array}{c}\text { Outpatients } \\
\text { No. }(\%)\end{array}$ & $\begin{array}{c}\text { Inpatients } \\
\text { No. }(\%)\end{array}$ \\
Wound & 46 & $15(32.6)$ & $31(67.4)$ \\
Sputum & 2 & $0(0)$ & $2(100)$ \\
\hline Aspirate & 3 & $0(0)$ & $3(100)$ \\
Blood & 12 & $4(33.3)$ & $8(66.7)$ \\
\hline Swabs & 18 & $7(38.9)$ & $11(61.1)$ \\
Cerebrospinal fluids & 2 & $0(0)$ & $2(100)$ \\
Urine & 1 & $0(0)$ & $11(100)$ \\
Total & 84 & $26(31.0)$ & $58(69.0)$ \\
\hline
\end{tabular}

Distribution of 84 methicillin-resistant Staphylococcus aureus (MRSA) isolates recovered from outpatients and inpatients. In patients had the highest carriage of MRSA representing $69 \%$, whiles out patients had a total of 26 isolates representing $31 \%$.

Table 3. Antibiotic percentage susceptibility profile of methicillin-resistant Staphylococcus aureus $(\mathbf{n}=84)$.

\begin{tabular}{lcc}
\hline ANIIBIOTICS & $\begin{array}{c}\text { Sensitive } \\
\text { No. (\%) }\end{array}$ & $\begin{array}{c}\text { Resistant } \\
\text { No. (\%) }\end{array}$ \\
Penicillin (1 ug) & $4(4.8)$ & $80(95.2)$ \\
Ampicillin(10 ug) & $13(15.5)$ & $71(84.5)$ \\
\hline Flucloxacillin (5 ug) & $24(28.6)$ & $60(71.4)$ \\
Erythromycin (5 ug) & $63(75.0)$ & $21(25.0)$ \\
Tetracycline (10 ug) & $6(7.1)$ & $78(92.8)$ \\
Cotrimoxazole (25 ug) & $35(41.7)$ & $49(58.3)$ \\
\hline Cefuroxime (30 ug) & $33(39.3)$ & $51(60.7)$ \\
Gentamicin (10 ug) & $46(54.7)$ & $38(45.2)$ \\
\hline
\end{tabular}

Resistant and sensitivity patterns of methicillin-resistant Staphylococcus aureus (MRSA) to various antibiotics. Penicillin recorded the highest resistance (80) to MRSA, whiles erythromycin showed least (21). 
agreed with the work done by Taiwo et al. ${ }^{22}$ The difference in the resistance of these $\beta$-lactam antibiotics is explained by the fact that PBP 2abinding affinities differ among the various $\beta$ lactam antibiotics; however, there is crossresistance to this general class of antibiotics because most, if not all, of the currently available agents bind too poorly to be active at clinically relevant concentrations. ${ }^{23}$

MRSA isolates also showed resistance as follows: tetracycline $92.8 \%$, and cotrimoxazole $58.3 \%$ (Table 3). High resistance of MRSA to gentamicin has also been reported ${ }^{24}$ However, in this study only $45.2 \%$ resistance to gentamicin was found. Furthermore, in another study, conducted among the aminoglycosides, findings revealed that susceptibility was maximum to netilmicin $52.5 \%$ and minimum to gentamicin $10.3 \%$ (showing that MRSA was $90 \%$ resistant to gentamicin). ${ }^{25}$ However, because of various reasons, netilmicin cannot be recommended for empirical treatment of MRSA associated infections. ${ }^{26}$ Lyon et al. have also shown that the majority of MRSA strains are also resistant to erythromycin, clindamycin, and tetracycline. ${ }^{27}$

\section{Conclusions and Recommendations}

First, the findings of this research have shown that there is an increasing prevalence rate of MRSA in Accra, Ghana. To reduce the prevalence of MRSA, regular surveillance of hospital associated infections, monitoring of antibiotic sensitivity patterns of MRSA and formulation of definite antibiotic policy may be helpful. There is therefore the need for health policy planners to be in the forefront in the development of comprehensive programs, policies and strategic measures as well as the development of research and surveillance units. It is recommended that molecular characterization is done in further studies.

\section{References}

1. Engemann JJ, Carmeli Y, Cosgrove SE, et al. Adverse clinical and economic outcomes attributable to methicillin resistance among patients with Staphylococcus aureus surgical site infection. Clin Infect Dis 2003;36:592-8.

2. Shopsin B, Kreiswirth BN. Molecular epidemiology of methicillin-resistant Staphylococcus aureus. Emerg Infect Dis 2001;7:1-8.
3. Scragg JN, Appelbaum PC, Govender DA. The spectrum of infection and sensitivity of organisms isolated from African and Indian children in a Durban hospital. Trans R Soc Trop Med Hyg 1978;72:325-8.

4. Geyid A, Lemeneh Y. The incidence of methicillin resistant Staphylococcus aureus strains in clinical specimens in relation to their beta-lactamase producing and multiple-drug resistance properties in Addis Ababa. Ethiop Med J 1991;29:149-61.

5. Omari MA, Malonza IM, Bwayo JJ, et al. Pattern of bacterial infections and antimicrobial susceptibility at the Kenyatta National Hospital, Nairobi, Kenya. East Afr Med J 1997;74:134-7.

6. Rotimi V0, Orebamjo OA, Banjo T0, et al. Occurrence and antibiotic susceptibility profiles of methicillin-resistant Staphylococcus aureus in Lagos University Teaching Hospital. Cent Afr J Med 1987;33: 95-9.

7. Okesola A0, Oni AA, Bakare RA. Prevalence and antibiotic sensitivity pattern of methicillin-resistant Staphylo-coccus aureus in Ibadan, Nigeria. J Hosp Infect 1999;41:74-5.

8. Sow AI, Wade A, Faye-Niang MA, et al. Methicillin-resistant Staphylococcus aureus in Dakar. Med Trop (Mars) 1998;58:155-7.

9. Gardee Y, Kirby R. The incidence of inducible macrolide-lincosamide-streptogramin B resistance in methicillinresistant staphylococci in clinical isolates from the Eastern Cape area of South Africa. Lett Appl Microbiol 1993;17:264-8

10. Van den Ende J, Rotter ML. An analysis of blood culture isolates from 7 South African teaching hospital centers. S Afr Med J 1986;69:89-93.

11. Peddie EF, Donald PR, Burger PJ, Sadler CA. Methicillin-resistant Staphylococcus aureus at Tygerberg Hospital. S Afr Med J 1988;74:223-4.

12. Musa HA, Shears P, Khagali A. First report of MRSA from hospitalized patients in Sudan. J Hosp Infect 1999;42:74.

13. Roman RS, Smith J, Walker M, et al. Rapid geographic spread of a methicillin-resistant Staphylococcus aureus strain. Clin Infect Dis 1997;25:698-705.

14. de Lencastre $H$, Aires-de Sousa M, Sanches I, et al. International spread of a multi-drug methicillin resistant Staphylococcus aureus clone. J Clin Microbiol 1998; $36: 2590-6$.

15. de Lencastre H, Melter O, Santo Sanches I, et al. Methicillin-resistant Staphylococcus aureus clonal types in the Czech Republic. J Clin Microbiol 1999;37:2798-803.
16. Mackenzie AMR, Richardson H, Lannigan R, Wood D. Evidence that the national committee for clinical laboratories standard disk test is less sensitive than the screen plate for detection of low-expression - class methicilin - resistant staphylococcus aureus. J Clin Microbiol 1995;33: 1909-11.

17. Felten A Grandry B Lagrange PH, Casin I. Evaluation of three techniques for detection of low-level methicilin resistant staphylococcus aureus (MRSA): a disk diffusion method with cefoxitin and maxalactam, the Vitek 2 system, and MRSA screen latex agglutination test. J Clin Microbiol 2002;40:2766-71.

18. Bowers KM, Wren MWD, Shetty NP. Screening for methicilin resistance in staphylococcus aureus and coagulase-negative staphylococci: an evaluation of three selective media and Mastlex-MRSA latex agglutination. Br J Biomed Sci 2003;60:714.

19. Atoum MF, Akel H, Battikhi MN. Comparison of PCR and disc diffusion method in detecting methicilin resistance among staphylococcus species from nosocomial infections. Saudi Med J 2003;24:1410-2.

20. Hall GS. MRSA: Lab detection, epidemiology, and infection control. Microbiology Frontline 2003;3:1-6.

21. Chambers HF. Methicillin resistance in staphylococci: molecular and biochemical basis and clinical implications. Clin Microbiol Rev 1997;10:781-91.

22. Taiwo S, Bamidel M, Omonigdehin E, et al. Molecular epidemiology of methicillin resistant Staphylococcus aureus in Ilorin, Nigeria. WAJM 2005;24:100-5.

23. Watanakunakorn C, Tisone JC. Synergism between vancomycin and gentamicin or tobramycin for methicillin-susceptible and methicillin- resistant Staphylococcus aureus strains. Antimicrob Agents Chemother 1982;22:903-5.

24. Anupurbas S, Sen MR, Nath G, et al. Prevelence of methicillin resistant staphylococcuo aureus in a tertiary referral hospital in eastern Uttar Pradesh. India J Med Microbiol 2003;21:49-51.

25. Bradley JN, None P. MRSA in a London hospital. Lancet 1985;1:1493-5.

26. Anupurba S, Sen MR, Nath G, et al. Prevalence of methicilin resistant staphylococcus aureus in tertiary referral hospital in eastern Uttar Pradesh. Ind J Med Microbiol Rev 1988;1:173-86.

27. Lyon BR, Skurray R. Antimicrobial resistance of staphylococcus aureus: genetic basis. Microbiol Rev 1987;51:88-134. 